\title{
Digital Image Analysis Using Immunohistochemistry for a Myoepithelial Marker (CK14/P63) Differentiating DCIS from Invasive Breast Cancer
}

\author{
Maeda I1*, Tajima S1, Noguchi A1, Chosokabe ${ }^{1}{ }^{1}$, Kojima ${ }^{2}$, \\ Kanemaki $\mathrm{Y}^{3}$, Ueno $\mathrm{T}^{4}$, Tsugawa $\mathrm{K}^{2}$ and Takagi $\mathbf{M}^{1}$ \\ ${ }^{1}$ Department of Pathology, St. Marianna University School of Medicine, Japan \\ ${ }^{2}$ Department of Breast and Endocrine Surgery, St. Marianna University School of \\ Medicine, Japan \\ ${ }^{3}$ Department of Radiology, St. Marianna University School of Medicine, Japan \\ ${ }^{4}$ Unit of Medical Statistics, St. Marianna University School of Medicine, Japan
}

\section{Research Article}

Volume 3 Issue 1

Received Date: February 23, 2019

Published Date: March 14, 2019

DOI: $10.23880 /$ cprj-16000114

*Corresponding author: Ichiro Maeda, St. Marianna University School of Medicine, 2-16-1 Sugao, Miyamae-ku, Kawasaki 216-8511, Japan, Email: ichirou@marianna-u.ac.jp

\section{Abstract}

Many researchers have reported immunohistochemistry of breast pathology studied using image-analysis software.

Aims: This study was to confirm the availability of digital image analysis (DIA) for breast pathology using immunohistochemistry for a myoepithelial marker with an antibody cocktail targeting CK14/p63 to differentiate ductal carcinoma in situ (DCIS) from invasive ductal cancer (IDC).

Methods and Material: We collected 139 consecutive core needle biopsy (CNB) specimens diagnosed as "malignant". All 139 CNB specimens were stained with a cocktail of antibodies targeting CK14/p63. All stained sections were photographed using data be scanned by a whole-slide imaging system. The images were analyzed using software to clarify the proportions of tumor cells that were positive or negative for the marker. The cutoff value for CK14/p63 (negative or positive) was determined using receiver operating characteristic (ROC) analysis.

Results: The cutoff value for CK14/p63 was 3.17\%, and a significant difference was found between DCIS and IDC (P < .0001). In DIA Dx, the sensitivity, specificity, positive predictive value for IDC, and negative predictive value for IDC were 76.9\%, 83.3\%, 93.9\%, and 34.9\%, respectively ( $\mathrm{P}<.0001)$.

Conclusion: DIA of immunostaining for CK14/p63 of breast cancer specimens is useful for distinguishing between DCIS and IDC.

Keywords: Breast; High Molecular; Myoepithelial cells; Fibroadenoma; Synaptophysin 
Abbreviations: DCIS: Ductal Carcinoma in Situ; IDC: Invasive Ductal Carcinoma; HMWCK: High-MolecularWeight Cytokeratin; DIA: Digital Image Analysis; ROI: Regions of Interest; CAD: Computer-Aided Diagnosis; HE: Hematoxylin and Eosin; VAB: Vacuum-Assisted Biopsy; WSI: Whole Slide Imaging AUC: Area Under Curve; ROC: Receiver Operating Characteristic; LR: Low-Risk; CNB: Core Needle Biopsy; US: Ultrasound; PNG: Portable Network Graphics.

\section{Introduction}

Ductal carcinoma in situ (DCIS) is considered to be a precursor lesion of invasive ductal carcinoma (IDC) and is known to progress to IDC in $14-53 \%$ cases if untreated [1]. Rane SU, et al. [2] has reported that copy number alteration of DCIS was increased similar to that of IDC. However, IDC and DCIS are considered to be two distinct classifications, because IDC is a systemic lesion and DCIS is a lesion local to the breast alone. In recent years, it has been thought that treatment for DCIS is being performed unnecessarily, a result of so-called "overdiagnosis" [3]. Furthermore, the concept of "low-risk (LR) DCIS" is being established. A study known as LORIS was started, and it found that a proportion of the patients having LR DCIS may not need surgical treatment [4]. In the LORIS study, the characteristics of LR DCIS are low- and intermediatenuclear-grade DCIS excluding high-nuclear-grade DCIS. There may be a problem that ultimately DCIS will be diagnosed using CNB materials, because the possibility that patients have IDC cannot be completely excluded. In the LORIS study, it was important that breast cancers were divided into low- and intermediate-grade DCIS or high-grade DCIS; however, it may be more important to differentiate DCIS and IDC. It has been reported that 36$40 \%$ of the diagnosis of DCIS in CNB materials is upgraded to IDC [5-8]. It is necessary to construct new methods to differentiate DCIS and IDC using other than the apparent morphology in histological diagnosis using CNB.

The presence or absence of myoepithelial cells is important in distinguishing DCIS from IDC of the breast. It has been reported that myoepithelial cells could be detected by antibodies against markers including p63 and CK14 [9]. Furthermore, Ichihara et al. have described the use of an antibody cocktail targeting p63 and highmolecular-weight cytokeratin (HMWCK) to differentiate borderline papillary lesions of the breast [10]. It has also been reported that use of a cocktail of antibodies targeting p63 and CK14 can distinguish intraductal papilloma [11] and fibroadenoma [12] from DCIS of the breast. Recently, computer-aided detection (CADe) of the presence or absence of lesions has been developed for radiology [13-15], and computer-aided diagnosis (CADx) for distinguishing benign from malignant lesions radiologically has been reported [15]. In breast pathology, digital image analysis (DIA) is beginning to be used; for example, for analysis of the Ki67 labeling index. Christgen et al. have reported that the setting of regions of interest (ROI) on histological images of Ki67 immunoreactivity in breast cancer is important, because the Ki-67 labeling index is greatly dependent on the ROI setting [16]. We have reported that we can calculate numerical values that do not depend so much on the ROI setting by including a so-called "hot spot" for Ki-67 immunoreactivity [17]. We have also reported that computer-aided diagnosis (CADx) of breast pathology using immunohistochemistry results of core needle biopsy samples for synaptophysin, estrogen receptor, and CK14/p63 are effective for classification of epithelial proliferative lesions of the breast [18]. In those studies, we found using a limited number of conditions that the CADx of breast pathology was not useful for distinguishing DCIS from IDC. To the best of our knowledge, no study has differentiated DCIS and IDC using DIA. The aim of this study was to clarify whether it is possible to distinguish between DCIS and IDC of the breast using DIA for immunohistochemistry targeting CK14/p63.

\section{Materials and Methods}

Calcification, cystic lesions, and mass lesions (hypoechoic lesions) detected by mammography and ultrasonography of the mammary gland in patients who underwent CNB at St. Marianna University Hospital from January 2013 to March 2013 were diagnosed as "malignant" by at least two pathologists who viewed slides stained with only hematoxylin and eosin (HE). CNB was performed using 14G stereotactic vacuum-assisted biopsy (VAB) of the breast, 11G ultrasound (US)-guided $\mathrm{VAB}$ of the breast, or 8G magnetic resonance imagingguided VAB of the breast.

\section{Definition of Final Diagnosis}

In the present study, we defined the ultimate histological classifications, DCIS or IDC, as histologically diagnosed in an open biopsy or (partial) mastectomy.

\section{Immunohistochemical Staining}

Four-micrometer-thick slices of representative blocks were prepared, placed on silane-coated glass slides, and stained in an automated immunostainer (Histostainer 36 A, Nichirei Biosciences, Tokyo, Japan). The primary antibody was a SqCC antibody cocktail (CK14/p63) 
(antibody cocktail of CK14 and p63; Pathology Institute, Toyama, Japan; dilution 1:1) (Table 1). All CNB specimens were immunohistochemically stained for CK14/p63.

\begin{tabular}{|c|c|c|c|c|}
\hline Markers & Company & Clone & Dilution & Antigen retrieval \\
\hline CK14/p63 & Pathology Institute & Cocktail antibody of CK14 and p63 & Pre-diluted & Antigen Retrieval Solution pH9 \\
\hline
\end{tabular}

Table 1: Antibodies for immunohitochemical analysis.

\section{Photographs}

For all of the samples, sections immunohistochemically stained with the antibody cocktail for CK14/p63 were scanned using a Whole Slide Imaging (WSI) system (VS120-L100, Olympus, Tokyo, Japan) using a $\mathrm{x} 40$ objective lens. All of these WSIs were imaged using a x20 field with an inset as a measure "50 um" [1747 x 871 pixels, PNG (Portable Network Graphics)].

\section{Image Analysis Software (Patholoscope) for DIA}

CK14/p63 immunoreactivity was analyzed in the nuclei and cytoplasm using a Patholoscope (Mitani Corp., Fukui, Japan). The Patholoscope was set as follows: cell size, 13 pixels; staining concentration, 17; extraction of immunonegative cells, 27; extraction of immunopositive cells, 12; measurement area, automation 75; deletion of small particles, 24; and deletion of elongated particles, 68 pixels.

\section{PDx}

In this study, the pathologists' diagnoses (PDx) were DCIS or IDC using only HE-stained sections assessed by at least two pathologists. Results of PDx were confirmed by final diagnosis as a reference.

\section{DIA Dx}

The cutoff value was determined by area under curve (AUC) analysis using DIA data in images from the immunohistochemical study for CK14/p63 in this case series. Results of the DIA for breast pathology differentiating DCIS from IDC (DIA Dx) using these cutoff values were confirmed by final diagnosis as a reference.

\section{PDx with DIA Dx}

PDx with DIA Dx was that the cases of diagnoses as DCISs by PDx were verified using DIA Dx. Results of PDx with DIA Dx were verified with final diagnosis as a reference.

\section{Statistical Analysis}

The AUC for the CK14/p63 antibody cocktail immunoreactivity was calculated by receiver operating characteristic (ROC) analysis. The statistical significance of differences was determined using a Fisher exact probability test when indicated. Differences with $\mathrm{P}<0.05$ were considered to be significant.

\section{Ethics Approval}

The study was approved by the ethics committee of St. Marianna University (approval number: 1915).

\section{Results}

One hundred thirty-nine consecutive cases that were diagnosed as malignant were selected from 380 consecutive CNB specimens. The malignant lesions included 31 DCIS and 108 IDCs on CNB in PDx. The 31 cases of DCIS on CNB diagnosis included 13 IDCs on final diagnosis. The 108 IDCs on CNB diagnosis were ultimately all diagnosed as IDCs. In PDx of CNB of this study, the sensitivity, specificity, positive predictive value (PPV) for IDC, and negative predictive value (NPV) for IDC were $89.3 \%, 100 \%, 100 \%$, and $58.1 \%$, respectively $(\mathrm{P}<.0001)$ (Table 2).

\begin{tabular}{|c|c|c|c|c|c|c|c|c|c|c|}
\hline \multicolumn{9}{|c|}{ Final diagnosis } \\
\hline & & DCIS & IDC & total & sensitivity & specificity & PPV for DCIS & NPV for DCIS & p value \\
\hline \multirow{2}{*}{ PDx } & DCIS & 18 & 13 & 31 & $100.0 \%$ & $89.3 \%$ & $58.1 \%$ & $100.0 \%$ & $<0.001$ \\
\cline { 2 - 12 } & IDC & 0 & 108 & 108 & & & & & \\
\hline \multirow{2}{*}{ CAIA Dx (CK14/p63) } & positive & 15 & 28 & 43 & $83.3 \%$ & $76.9 \%$ & $34.9 \%$ & $96.9 \%$ & $<0.001$ \\
\cline { 2 - 11 } & negative & 3 & 93 & 96 & & & & & \\
\hline \multirow{2}{*}{ PDx with CAIA Dx } & DCIS & 15 & 7 & 22 & $83.3 \%$ & $94.2 \%$ & $68.2 \%$ & $97.4 \%$ & $<0.001$ \\
\cline { 2 - 10 } & IDC & 3 & 114 & 117 & & & & & \\
\hline
\end{tabular}

Table 2: Sensitivity, specificity, positive predictive value for DCIS, and negative predictive value for DCIS for CAIA, PDx, and PDx with CAIA Dx. 
The cutoff values of CK14/p63 for distinguishing DCIS from IDC were determined by using the AUC for an antibody, as computed by ROC analysis. The cutoff value for CK14/p63 was $3.17 \%$ in this study. The malignant lesions included 43 DCIS (Figures 1(a-c)) and 96 IDCs (Figures 2(a-c)) on CNB diagnosis using DIA for CK14/p63. The 43 cases of DCIS in DIA Dx included 28 IDCs on final diagnosis, and the 96 IDCs in DIA Dx included three cases of DCIS on final diagnosis. In DIA Dx of the present study, the sensitivity, specificity, PPV for IDC, and NPV for IDC were $76.9 \%, 83.3 \%, 93.9 \%$, and $34.9 \%$, respectively $(\mathrm{P}<.0001)$ (Table 2$)$. In PDx with DIA Dx, the sensitivity, specificity, PPV for IDC, and NPV for IDC were $94.2 \%, 83.3 \%, 95.0 \%$, and $68.2 \%$, respectively $(\mathrm{P}<.0001)$ (Table 2).
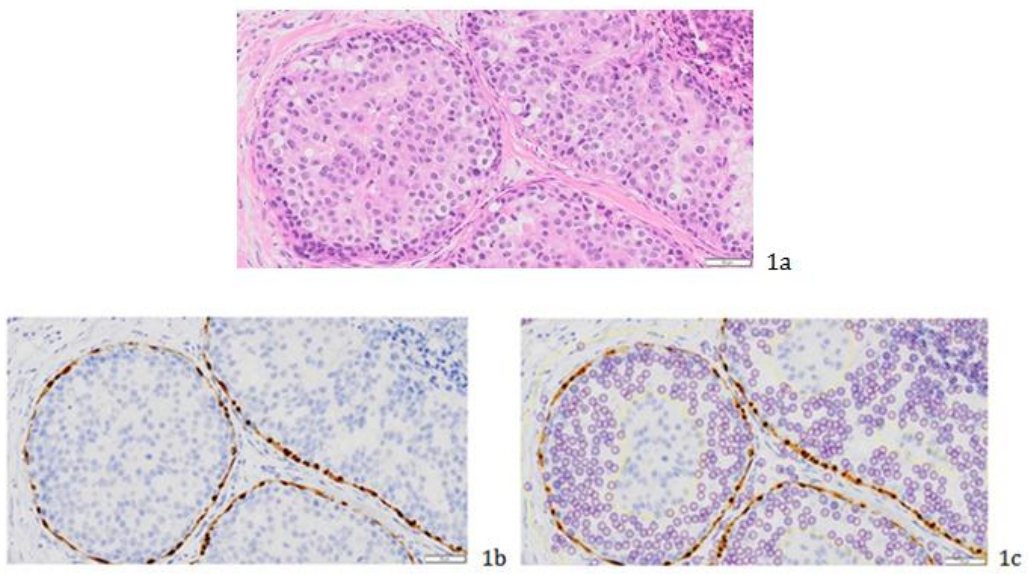

Figure 1(a-c): 1a: DCIS case, 1b: HE staining, 1c: immunohistochemical staining for CK14/p63; analyzed imaging using image analysis software. The orange rings indicate immunopositive cells, and the blue rings indicate immunonegative cells.

The yellow lines surround the lesions. Almost all of the and the cancer cells are immunonegative for CK14 and myoepithelial cells are immunopositive for CK14 and p63, p63 (1c).
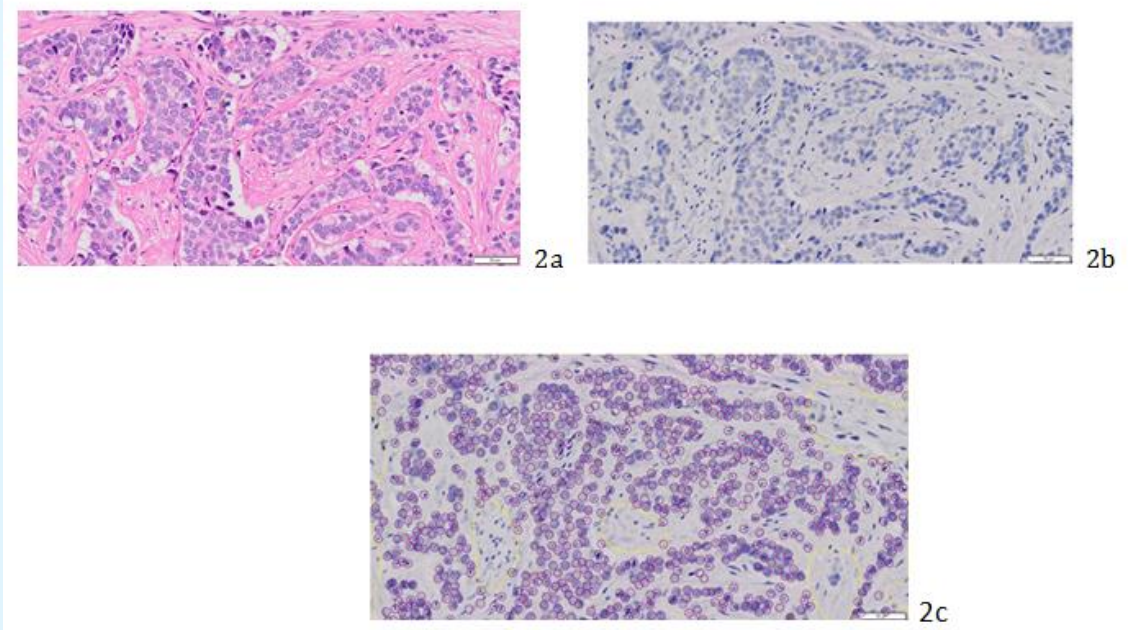

Figure 2(a-c): 2a: Invasive ductal carcinoma case, 2b: HE staining, 2c: immunohistochemical staining for CK14/p63, analyzed imaging using image analysis software. Almost all cancer cells are immunonegative for CK14 and p63. Immunopositive cells are not found (2c). 


\section{Discussion}

In the present study, DIA Dx of immunohistochemistry for CK14/p63 was useful for differentiating DCIS from IDC (P<.0001), and PPV for IDC of DIA Dx and PDx was 93.9\% and $100 \%$, respectively. Moreover, NPV for IDC was increased to $68.2 \%$ when DIA Dx was used for cases of DCIS that a pathologist diagnosed (PDx with DIA Dx). No significant difference was found between DCIS of final diagnosis and IDC of final diagnosis for the cases of DCIS found by $\mathrm{PDx}(\mathrm{P}=0.3316$, data not shown $)$.

In fine needle biopsy specimens of the breast, the presence of myoepithelial cells has differentiated DCIS from IDC [19], DCIS from fibroadenoma [12], and DCIS from intraductal adenoma [11]. These studies have indicated that the numbers of myoepithelial cells were decreased in DCIS more than they were in benign lesions. The present study found that myoepithelial cells were less frequent in IDC than in DCIS. This may indicate that the probability of a malignancy increases with decreasing frequency of myoepithelial cells. Cancer cells are influenced by paracrine regulators from the host microenvironment, and normal duct cells or cancer cells may be influenced by paracrine regulators from myoepithelial cells [20]. Myoepithelial cells regulate increased angiogenesis, proliferation of fibroblasts and myofibroblasts, and accumulation of extracellular matrix. In fact, myoepithelial cells are thought to constitute the microenvironment of a normal breast duct [21,22]. Decreased numbers of myoepithelial cells may promote cancer invasion by increased angiogenesis, proliferation of fibroblasts and myofibroblasts, and accumulation of extracellular matrix. Apoptosis of glandular epithelial cells is considered to be involved in glandular cavity formation [23]. Myoepithelial cells overexpress p63 and p73 [24]. P63 and p73 as part of the p53 family have numerous functions, and one of these functions is the control of apoptosis. Binding of p63, p73, and mutated p53 regulate p21 as a cyclin-dependent kinase inhibitor at the G1 phase of the cell cycle and apoptosis via PERP $[25,26]$. These mechanisms work in a complicated manner, creating a microenvironment. Decreased numbers of myoepithelial cells may promote cancer invasion because of the disruption of this microenvironment.

We have reported that CADx of breast pathology using immunohistochemistry for CK14/p63, synaptophysin, and estrogen receptor is effective for distinguishing benign epithelial proliferative lesions from malignant lesions. It may be more effective to combine DIA Dx and CADx for breast pathology to differentiate the benign lesion DCIS and IDC. Our results have proven this hypothesis. Yamamoto, et al. [27] have reported that characteristics of myoepithelial cells of DCIS were analyzed quantitatively using artificial intelligence (AI) with deep learning [27]. Adding analysis using AI to DIA Dx may be necessary, because PPV for DCIS using DIA Dx was not sufficient in the present study. The accuracy of differentiating between DCIS and IDC on the CNB diagnosis may be improved by adding DIA Dx analysis with AI. We face some challenges to the implementation of DIA. For cases immunonegative for CK14/p63, it was found that DIA Dx was able to distinguish DCIS from IDC $(\mathrm{P}<.0001)$. However, IDC was judged as DCIS in seven cases of DIA Dx in the present study. There are three possible causes as follows. First, in a few cases, final diagnosis was encapsulated papillary carcinoma (EPC) (Figures 3(a-c)). Collins LC, et al. [28] have reported that the myoepithelial layer could not be detected using immunohistochemistry for myoepithelial markers at the periphery of the nodules in cases of EPC, and Collins et al. have stated that "we believe it is most prudent to continue to manage patients with these lesions as they are currently managed (i.e., similar to patients with DCIS) and to avoid categorization of such lesions as frankly invasive papillary carcinomas", whereas other researchers have reported finding lymph node metastasis in patients with EPC $[28,29]$. Based on the observations of Collins et al. and the results of the present study, we consider that patients with EPC should be treated as having microinvasive carcinoma. Second, numbers of myoepithelial cells were severely decreased in some cases of DCIS (Figures $4(\mathrm{a}-\mathrm{c})$ ).

In cases of DCIS, myoepithelial cells were less prevalent than in adjacent normal breast ducts as determined by immunohistochemical staining for CK14, p63, and calponin [30]. Some researchers have reported that myoepithelial cells are less prevalent in cases of DCIS than in cases of IDP or FA $[11,12,31]$. Patients with DCIS and a dramatic decrease in the number of myoepithelial cells may border on having IDC, perhaps because of the disruption of the microenvironment as a result of the loss of two layers of cells around the ducts. Third, in a few cases, there was a problem with the range of the imaging; for example, in the image of cancer cells that excludes the myoepithelial layer (Figures 5(a-c)). In the present study, such images acquired from a portion of the central zone of the DCIS were analyzed. This problem may be solved by analyzing images using a WSI system. 

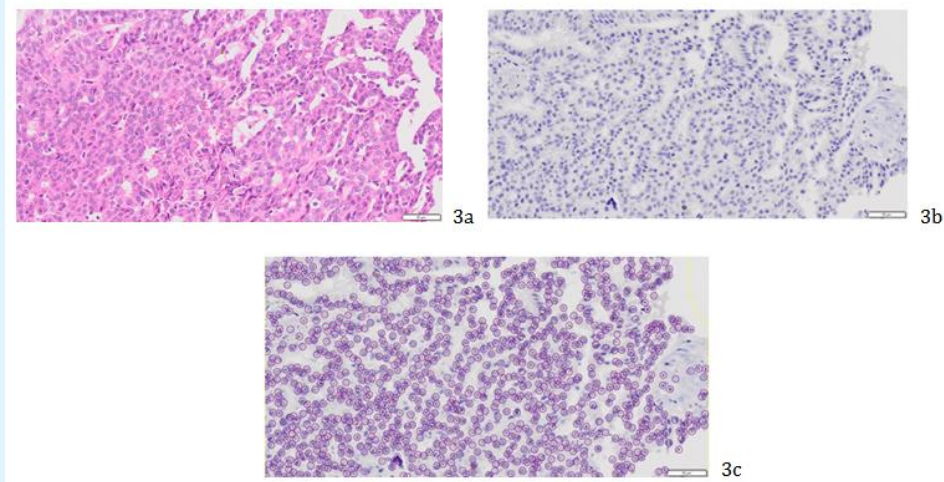

Figure 3(a-c): 3a: Encapsulated papillary carcinoma case, 3b: HE staining, 3c: immunohistochemical staining for CK14/p63; analyzed imaging using image analysis software. Almost all cancer cells are immunonegative for CK14 and p63. Immunopositive cells are not found (3c).
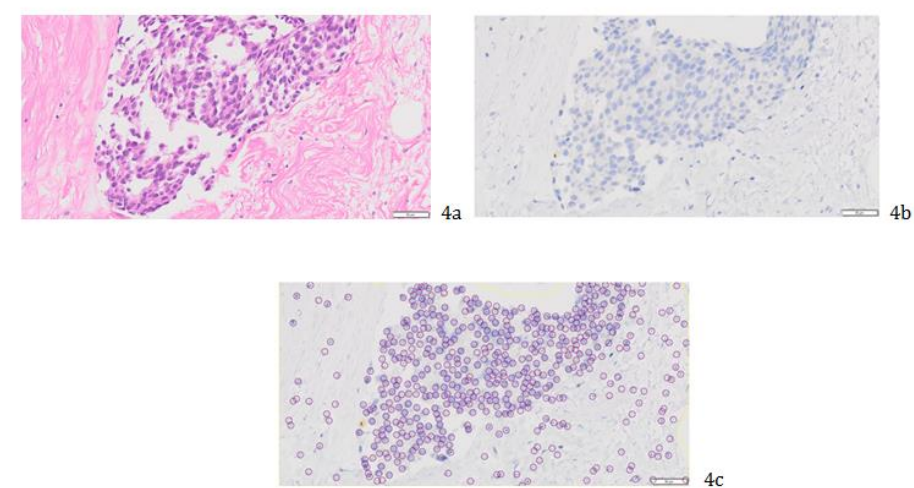

Figure 4(a-c): 4a: DCIS case, 4b: HE staining, 4c: immunohistochemical staining for CK14/p63; analyzed imaging using image analysis software. Almost all cancer cells are immunonegative for CK14 and p63. An immunopositive cell is seen $(4 c)$.
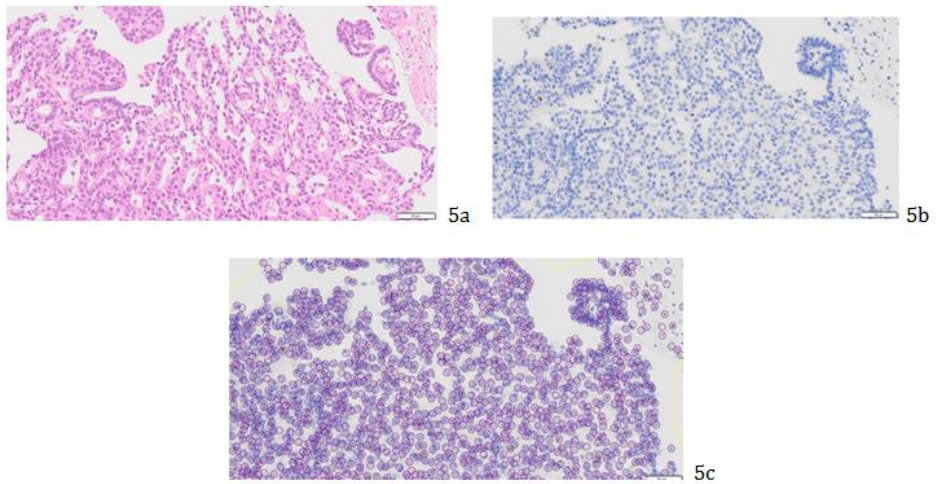

Figure 5(a-c): 5a: DCIS case, 5b: HE staining, 5c: immunohistochemical staining for CK14/p63; analyzed imaging using image analysis software. Almost all cancer cells were immunonegative for CK14 and p63. A stromal area around DCIS and immunopositive cells is not found (5c). 
Advantage of DIA is thought that digital diagnosis using DIA might speculate invasive carcinoma of final diagnosis by the case of DCIS on morphology. Furthermore, DIA is a good new tool in present days. And DIA might helpful tool for pathologists, because DIA was able to diagnose without concerning any doctors. There are limitations of this research as follows. First, analyzed images included only parts of CNB sections. Second, a validation study was not performed. Using this cutoff point, multicenter collaborative research should be needed in the future. Third, only CK14 and p63 myoepithelial markers were demonstrated, without smooth muscle actin, smooth muscle myosin heavy chain, CD10, calponin, or p75 [32]. The diagnosis after surgery by multi-focal sampling, and long-term follow-up data will be helpful in supporting the current results. The limitation of the methods are first, the DIA machine of the capability, second the number of cases is not enough, so multicenter collaborative research might be needed and the increase the number of cases.

\section{Conclusion}

DIA of immunostaining for CK14/p63 of breast cancer specimens is useful for distinguishing between DCIS and IDC.

\section{Acknowledgment}

We are grateful to Manabu Kubota for scanning in a whole-slide imaging and checked the imaging of all whole-slide imaging. We thank Jiro Ohta for setting parameter of image analysis software. This study was supported by grants from the Japan Agency for Medical Research and Development (No. 16lk1010007h0001 and No. 17lk1010006h9902).

\section{Author Contributions}

IM: Conceptualization, methodology, formal analysis, investigation, data curation, writing-original draft, writing-review, project administration, and funding for acquisition. ST: Conceptualization, investigation, writingreview and editing. AN, MC, and YK: Writing-review and editing. TU: Conceptualization, methodology, and formal analysis. KT, YK, and MT: Review.

\section{Disclosure/Conflict of Interest Statement}

The authors have nothing to disclose.

\section{References}

1. Erbas B, Provenzano E, Armes J, Gertig D (2006) The natural history of ductal carcinoma in situ of the breast: a review. Breast Cancer Res and Treat 97(2): 135-144.

2. Rane SU, Mirza H, Grigoriadis A, Pinder SE (2015) Selection and evolution in the genomic landscape of copy number alterations in ductal carcinoma in situ (DCIS) and its progression to invasive carcinoma of ductal/no special type: a meta-analysis. Breast Cancer Res and Treat 153(1): 101-121.

3. Bleyer A, Welch HG (2012) Effect of three decades of screening mammography on breast-cancer incidence. N Engl J Med 367: 1998-2005.

4. UK CR (2014) A trial comparing surgery with active monitoring for low risk DCIS (LORIS).

5. Usami S, Moriya T, Amari M, Suzuki A, Ishida T, et al. (2007) Reliability of prognostic factors in breast carcinoma determined by core needle biopsy. Jpn J Clin Oncol 37(4): 250-255.

6. Wei X, Li Y, Zhang S, Zhu Y, Fan Y (2011) Experience in large-core needle biopsy in the diagnosis of 1431 breast lesions. Med Oncol 28(2): 429-433.

7. Brem RF, Schoonjans JM, Sanow L, Gatewood OM (2001) Reliability of histologic diagnosis of breast cancer with stereotactic vacuum-assisted biopsy. Am Surg 67(4): 388-392.

8. Burak WE Jr, Owens KE, Tighe MB, Kemp L, Dinges SA, et al. (2000) Vacuum-assisted stereotactic breast biopsy: histologic underestimation of malignant lesions. Arch Surg 135(6): 700-703.

9. Tang P, Tse GM (2016) Immunohistochemical Surrogates for Molecular Classification of Breast Carcinoma: A 2015 Update. Arch Pathol Lab Med 140(8): 806-814.

10. Ichihara $\mathrm{S}$, Fujimoto $\mathrm{T}$, Hashimoto $\mathrm{K}$, Moritani $\mathrm{S}$, Hasegawa M, et al. (2007) Double immunostaining with p63 and high-molecular-weight cytokeratins distinguishes borderline papillary lesions of the breast. Pathol Int 57(3): 126-132.

11. Oana Y, Maeda I, Fukushima M, Tsugawa K, Takagi M (2015) Useful Double Immunostaining with p63 and CK14 in the Differential Diagnosis of IDP and DCIS in Fine Needle Aspiration Cytology of the Breast. The 
Journal of the Japanese Society of Clinical Cytology 54(2): 107-113.

12. Maeda I, Oana Y, Tsugawa K, Takagi M (2017) Availability of immunocytochemistry using cocktail antibody targeting p63/cytokeratin14 for the differential diagnosis of fibroadenoma and ductal carcinoma in situ in fine needle aspiration cytology of the breast. Cytopathology 28(5): 378-384.

13. Awai K, Murao K, Ozawa A, Komi M, Hayakawa H, et al. (2004) Pulmonary nodules at chest CT: effect of computer-aided diagnosis on radiologists' detection performance. Radiology 230(2): 347-352.

14. van Ginneken B, Schaefer-Prokop CM, Prokop M (2011) Computer-aided diagnosis: how to move from the laboratory to the clinic. Radiology 261(3): 719732.

15. Li Q, Li F, Suzuki K, Shiraishi J, Abe H, et al. (2005) Computer-aided diagnosis in thoracic CT. Semin Ultrasound CT MR 26(5): 357-363.

16. Christgen $M$, von Ahsen S, Christgen $H$, Langer $F$, Kreipe H (2015) The region-of-interest size impacts on Ki67 quantification by computer-assisted image analysis in breast cancer. Hum Pathol 46(9): 13411349.

17. Maeda I, Abe K, Koizumi H, Nakajima C, Tajima S, et al. (2016) Comparison between Ki67 labeling index determined using image analysis software with virtual slide system and that determined visually in breast cancer. Breast Cancer 23(5): 745-751.

18. Maeda I, Kubota M, Ohta J, Shinno K, Tajima S, et al. (2017) Effectiveness of computer-aided diagnosis (CADx) of breast pathology using immunohistochemistry results of core needle biopsy samples for synaptophysin, oestrogen receptor and CK14/p63 for classification of epithelial proliferative lesions of the breast. J clin Pathol 70(12): 1057-1062.

19. Reis-Filho JS, Milanezi F, Amendoeira I, Albergaria A, Schmitt FC (2002) p63 Staining of myoepithelial cells in breast fine needle aspirates: a study of its role in differentiating in situ from invasive ductal carcinomas of the breast. J clin Pathol 55(12): 936939.

20. Barsky SH, Karlin NJ (2005) Myoepithelial cells: autocrine and paracrine suppressors of breast cancer progression. J Mammary Gland Biol Neoplasia 10(3): 249-260.

\section{Clinical Pathology \& Research Journal}

21. Allen MD, Marshall JF, Jones JL (2014) $\alpha v \beta 6$ Expression in myoepithelial cells: a novel marker for predicting DCIS progression with therapeutic potential. Cancer Res 74(21): 5942-5947.

22. Polyak K, Hu M (2005) Do myoepithelial cells hold the key for breast tumor progression?. J Mammary Gland Biol Neoplasia 10(3): 231-247.

23. Mills KR, Reginato M, Debnath J, Queenan B, Brugge JS (2004) Tumor necrosis factor-related apoptosisinducing ligand (TRAIL) is required for induction of autophagy during lumen formation in vitro. Proc Natl Acad Sci U S A 101(10): 3438-3443.

24. Yamamoto $\mathrm{T}$, Oda $\mathrm{K}$, Miyazaki $\mathrm{K}$, Ichigotani $\mathrm{Y}$, Takenouchi Y, et al. (2001) p73 is highly expressed in myoepithelial cells and in carcinomas with metaplasia. Int J Oncol 19(2): 271-276.

25. Li Y, Prives C (2007) Are interactions with p63 and p73 involved in mutant p53 gain of oncogenic function? Oncogene 26(15): 2220-2225.

26. Awais R, Spiller DG, White MR, Paraoan L (2016) p63 is required beside p53 for PERP-mediated apoptosis in uveal melanoma. Br J Cancer 115(8): 983-992.

27. Yamamoto Y, Saito A, Tateishi A, Shimojo H, Kanno H, et al. (2017) Quantitative diagnosis of breast tumors by morphometric classification of microenvironmental myoepithelial cells using a machine learning approach. Sci Rep 7: 46732.

28. Collins LC, Carlo VP, Hwang H, Barry TS, Gown AM, et al. (2006) Intracystic papillary carcinomas of the breast: a reevaluation using a panel of myoepithelial cell markers. Am J Surg Pathol 30(8): 1002-1007.

29. Rakha EA, Gandhi N, Climent F, van Deurzen CH, Haider SA, et al. (2011) Encapsulated papillary carcinoma of the breast: an invasive tumor with excellent prognosis. Am J Surg Pathol 35(8): 10931103.

30. Rohilla M, Bal A, Singh G, Joshi K (2015) Phenotypic and Functional Characterization of Ductal Carcinoma In Situ-Associated Myoepithelial Cells. Clin Breast Cancer 15(5): 335-342.

31. Tanaka S, Kanomata N, Teramura K, Wakita K, Kunihisa T, et al. (2006) Usefulness of immunocytochemistry using a Breast Marker antibody cocktail targeting P63/ cytokeratin 7/18/ cytokeratin 5/14 for fine needle aspiration of the breast: a 
retrospective cohort study of 139 cases. Cytopathology 27(6): 465-471.

32. Hilson JB, Schnitt SJ, Collins LC (2009) Phenotypic alterations in ductal carcinoma in situ-associated myoepithelial cells: biologic and diagnostic implications. Am J Surg Pathol 33(2): 227-232. 